المجلة العراقية للعلوم الإحصائية (30) 2019

ص ص [24-1]

المقدر التقريبي لمعلمات أنموذج VMA(1) غير الطبيعي

د."وصفي طاهر صالح"

د. ديفاء عبد الجواد سعيد"

haeifa965@gmail.com

د. دحاسن صالح عبدالله الطالب"

wasfit@gmail.com

msat563@yahoo.com

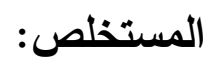

في كثير من تطبيقات السلاسل الزمنية يكون حد التشويش الأبيض لا يتبع التوزيع الطبيعي، بل

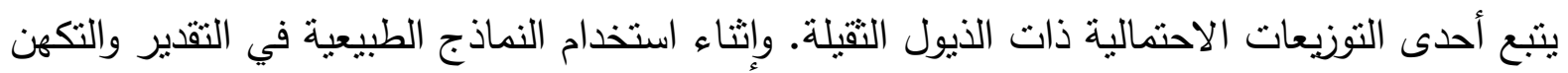
بتلك الظواهر ، فإنها سوف تتتج مقدرات وتكهنات بعيدة عن الواقع وغير كفؤة.

ينتمي توزيع بسل متعدد المتغيرات المحور المعدم ( Generalized multivariate (modified Bessel distribution تطبيقات واسعة في الظواهر التي تتغير عبر الزمن، وعلى هذا الأساس فقد تتاول البحث دراسة أنموذج

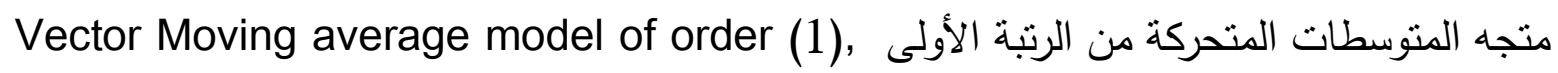

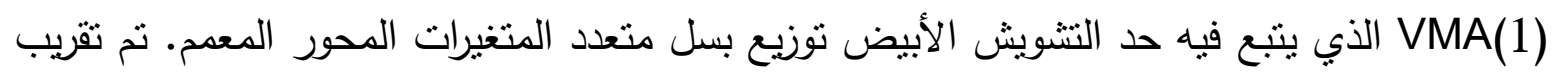

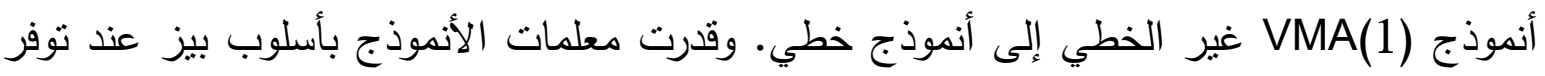

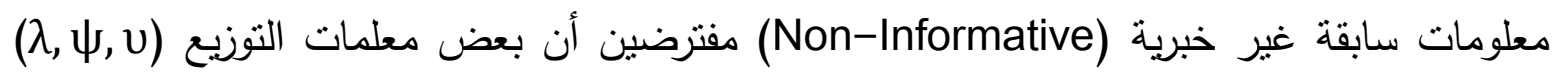
تكون معلومة. استخدمت في أسلوب بيز دوال خسارة مختلفة ، وهي التربيعية والأُسية الخطية

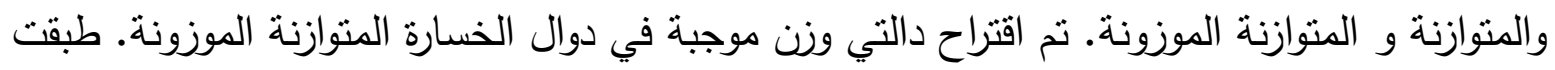

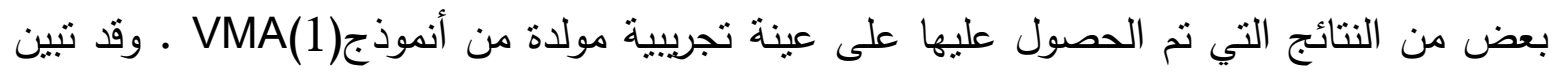

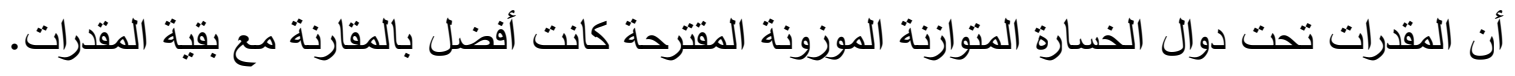

This is an open access article under the CC BY 4.0 license http://creativecommons.org/licenses/by/4.0/).

"مدرس/ قسم الاحصاء وإلمطلوماتية/ كلية الحاسوب والرياضيات/ جامعة الموصل

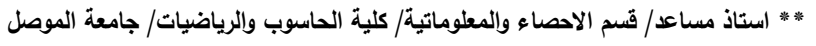

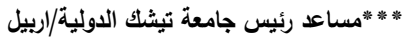

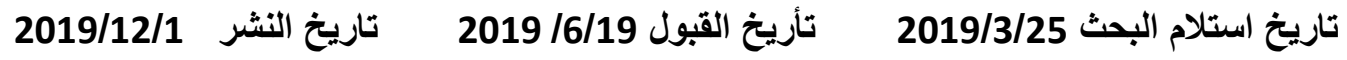




\section{Approximate estimator for parameters of non-normal VMA(1) model}

\section{Abstract:}

In many applications of time series, the white noise does not follow the normal distribution but follows one of the heavy tailed distributions. When

using normal models in estimation and forecasting, these phenomena they will produce far from reality and inefficient estimators and predictions. The generalized multivariate modified Bessel distribution belongs to the potentially heavy-tailed distribution family and has wide applications in events that change over time. On this basis, this paper concerned with the study of vector moving average model of the first order $(\operatorname{VMA}(1))$, is the white noise error term of this model follows GMMB. The non linear VMA(1) model was approximated to a linear model. The parameters of approximated model was estimated by Bayesian technique when non-informative priors. We supposed that some parameters of the distribution $(\lambda, \psi, v)$ known.

Different loss functions has been used in Bayesian analysis, We proposed two positive weight functions in weighted balanced loss functions .

Some of theoretical results were applied on empirical sample generated from VMA(1) model. It is concluded that the estimators under proposed weighted balanced loss functions are better.

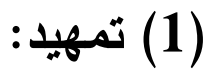

Generalized Multivariate متعدد المتغيرات المحور المعمم Bessel ينتمي توزيع Modified Bessel distribution (GMMB) التوزيع الأشمل من التوزيع الطبيعي وتوزيع t (Thabane and Haq(2003) وله تطبيقات واسعة في الظواهر التي تتغير عبر الزمن التي لها أهمية كبيرة في صنع القرار كقياس مقدرة وجودة العملية الإنتاجية وأسعار الأسهم المالية والسلع الأساسية ، فقد أصبحت أسعار الأسهر كمدخلات أساسية لآلية

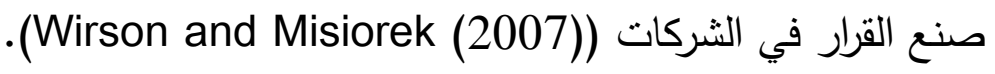

بتمتع توزيع GMMB بخاصية الخطية فقد استخدمه (العبيدي ، سعيد (2013)) كتوزيع

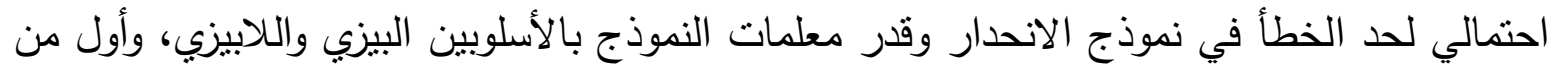

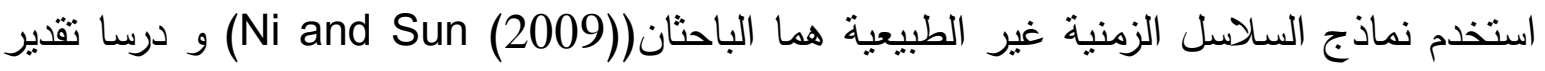

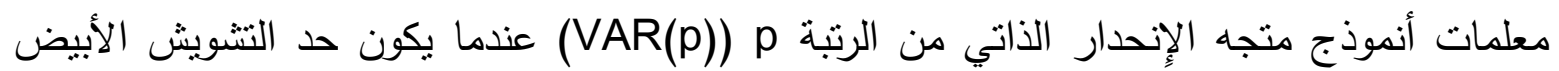


يتبع توزيع t المتعدد وقدر معلماته بأُسلوب بيز تحت دوال خسارة مختلفة ، وأشكال مختلفة للتوزيعات

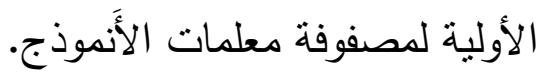
إن أنموذجي الأحادية المتغير، ومتعددة المتغيرات هي نماذج لاخطية في

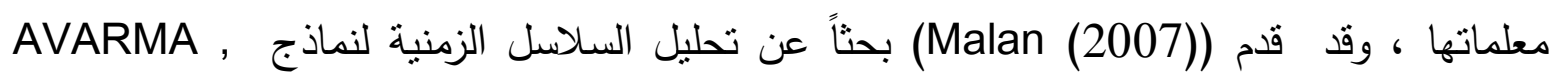

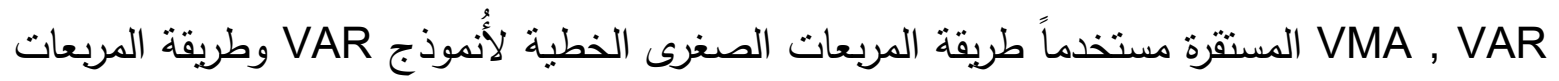

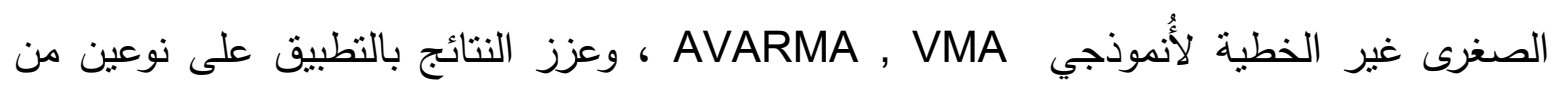
البيانات حقيقية ومولدة. وقدم (Fan and Yao) بحثاً وضحا فيه الأسلوب البيزي في تقدير معلمات نموذجي AVARMA , VMA الطبيعيين ، ولاقيا صعوبة في تحديد قيمة حد التشويش الأبيض فقد

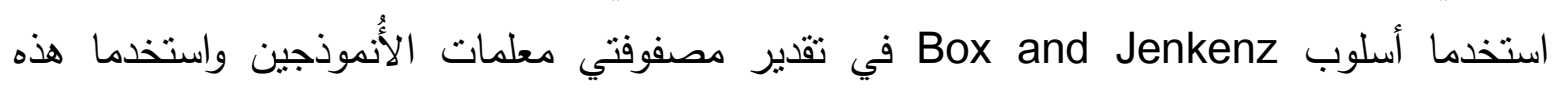
المقدرات في تقدير حد النشويش الأبيض، واعتبراها متجهات مشاهدات معلومة وباستخدام التوزيعات

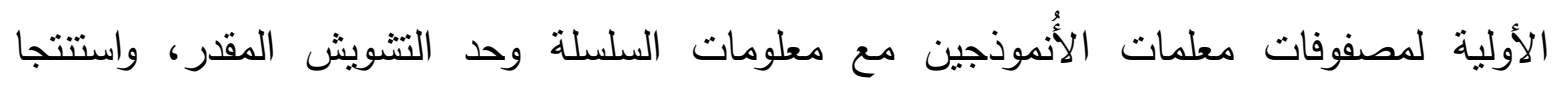

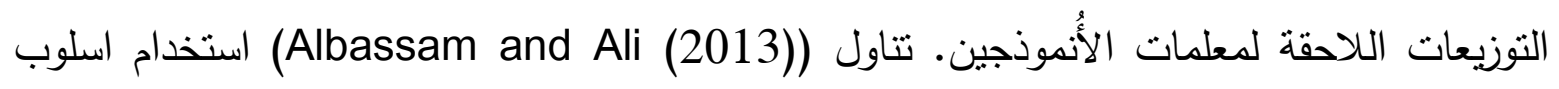
(بيز) غير المباشر في تحليل أنموذج المتوسطات المتحركة الطبيعي تحت ثلاثة أنواع من التوزيعات

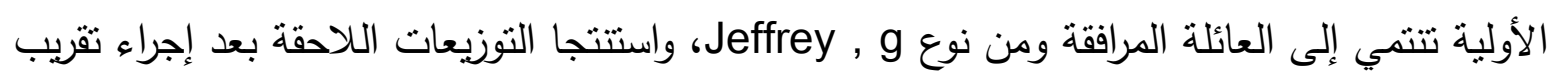
لدالة الإمكان غير الخطية، وتبين أن التوزيعات اللاحقة لمعلمات النماذج تتبع توزيع t غير المركزي

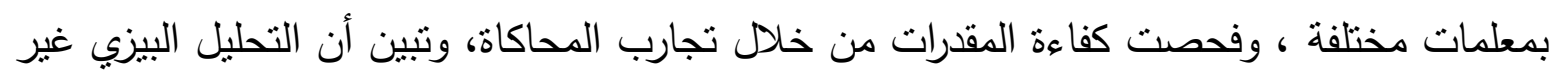
المباشر هو الأفضل في عملية التقدير.

استخدم (الطالب (2017)) أسلوباً قرب فيه أنموذج متجه المتوسطات المتحركة من الرتبة الأولى إلى أنموذج خطي الذي يكون فيه حد التتويش يتبع توزيع GMA(1) في تقدير معلماته تحت دوال خسارة مختلفة.

يضم المبح الثاني وصفاً لأُموذج VMA(1) ، والمبحث الثالث تتاول كيفية تقريب أنموذج

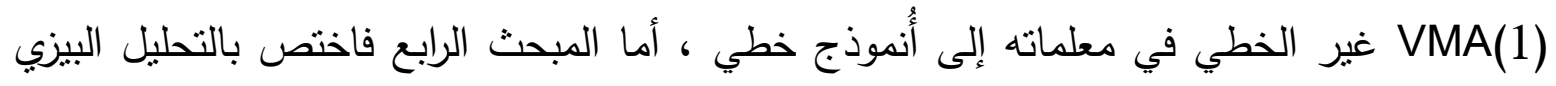

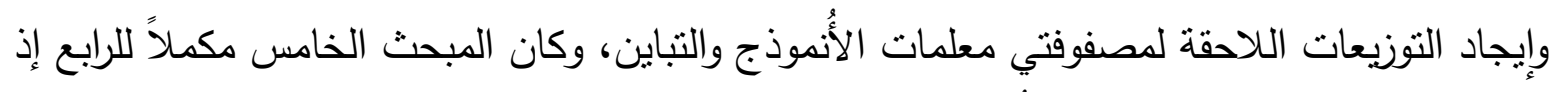

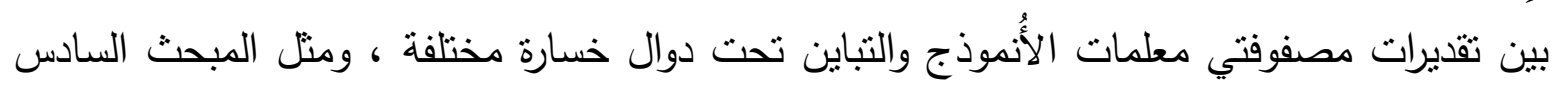

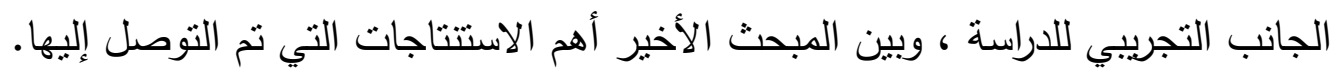


(2) (2) وصف الأُموذج:

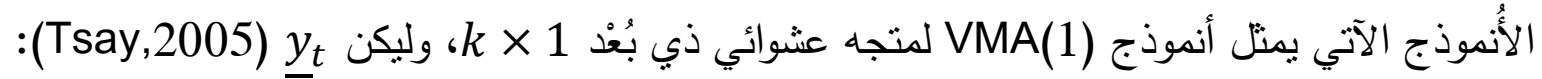
$\underline{y}_{t}=\underline{b}-\theta \underline{u}_{t-1}+\underline{u}_{t} \quad, \mathrm{t}=1,2, \ldots, \mathrm{T}$

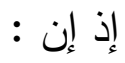

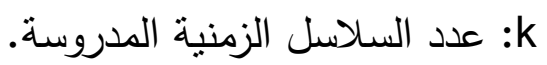

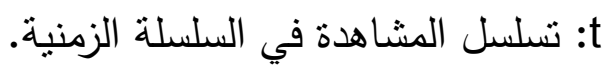

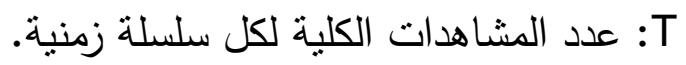

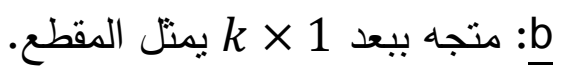

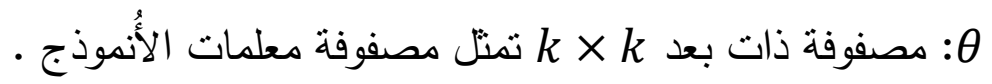
ألئ

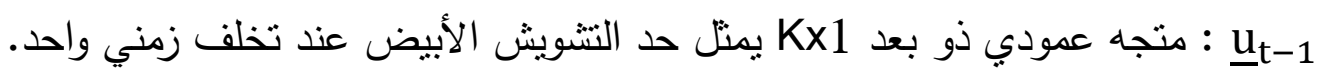
$\underline{\mathrm{y}}_{\mathrm{t}}=\left[\begin{array}{llll}\mathrm{y}_{1 \mathrm{t}} & \mathrm{y}_{2 \mathrm{t}} & \cdots & \mathrm{y}_{k \mathrm{t}}\end{array}\right]^{\prime}$

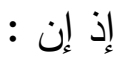

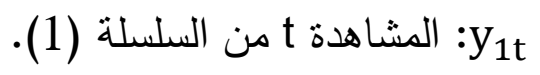

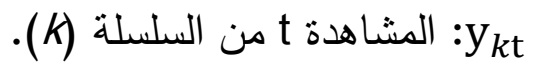

$\underline{\mathrm{u}}_{\mathrm{t}}=\left[\begin{array}{llll}\mathrm{u}_{1 \mathrm{t}} & \mathrm{u}_{2 \mathrm{t}} & \ldots & \mathrm{u}_{k \mathrm{t}}\end{array}\right]^{\prime}$

$\underline{b}=\left[\begin{array}{llll}b_{1} & b_{2} & \ldots & b_{k}\end{array}\right]^{\prime}$ وبفرض أن المقطع (bِ) متجه معلوم ، وعليه يمكن كتابة الأنموذج (1) بالصيغة الآتية : $\underline{y}_{t}^{*}=\underline{u}_{t}-\theta \underline{u}_{t-1}$

$\underline{y}_{t}^{*}=\underline{y}_{t}-\underline{b}$

و يمكن تمثيل حد التثويش الأبيض من الأنموذج (2) بالصيغة الآتية : $\underline{u}_{t}=\underline{y}_{t}^{*}+\theta \underline{u}_{t-1}$

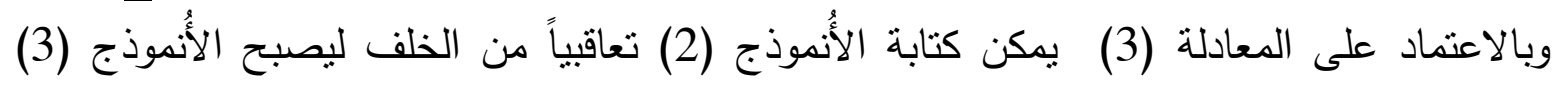
بالصيغة الآتية(Wei 2006): 
$\underline{y}_{t}^{*}=\underline{u}_{t}-\sum_{j=1}^{t-1} \theta^{j} \underline{y}_{t-j}$

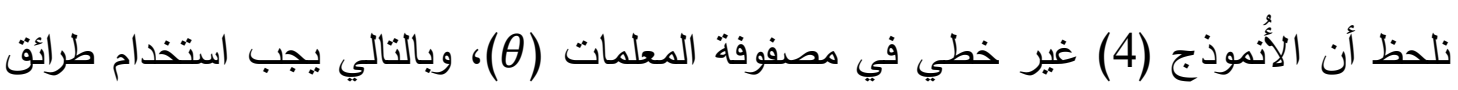

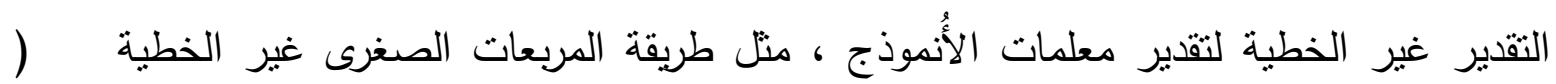

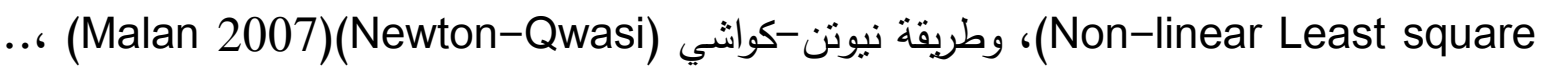
، ولما كانت هذه الطرائق لا يمكن استخدامها في التقدير البيزي فقد نم اقتراح أسلوب لتحويل الأنموذج إلى أنموذج خطي وذلك باستخدام مفكوك تايلر .

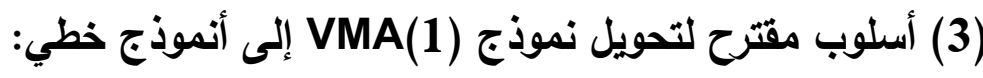

بالإمكان تقريب الأنموذج (4) إلى أنموذج خطي بالاعنماد على مفكوك تايلور، إذ يتم تقريب إنى الحد الثاني من الطرف الأيمن إلى خطي ، وذللك باعتماد الخطوات الآتية : نكتب المعادلة (4) بالصيغة الآتية:

$\underline{y}_{t}^{*}=\underline{u}_{t}-f(\theta)$

إذ إن

$f(\theta)=\sum_{j=1}^{t-1} \theta^{j} \underline{y}_{t-j}^{*}$

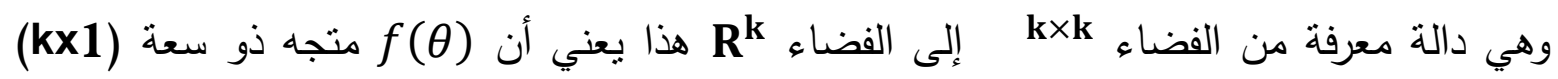
وقابلة للاشتقاق إذ يمكن أن تكتب بالصيغة الآتية :

$f(\theta)=\sum_{j=1}^{t-1} \operatorname{vec}\left(I_{k} \theta^{j} \underline{y}_{t-j}^{*}\right)$

و باستخدام خصائص عملية الـ Vector operator نحصل على:

$f(\theta)=\sum_{j=1}^{t-1}\left(\underline{y}_{t-j}^{* \prime} \otimes I_{k}\right) \operatorname{vec}\left(\theta^{j}\right)$

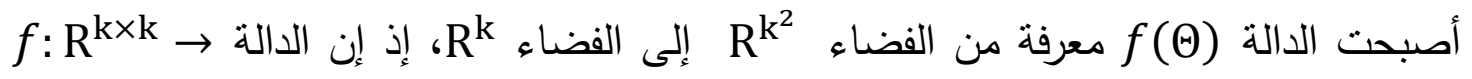
. $f: \mathrm{R}^{\mathrm{k}^{2}} \rightarrow \mathrm{R}^{\mathrm{k}}$ هي $\mathrm{R}^{\mathrm{k}}$

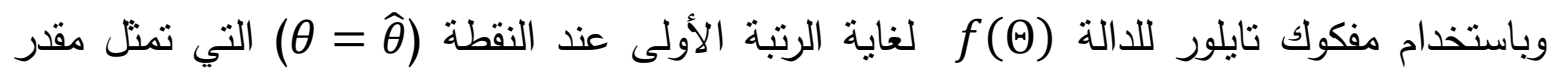

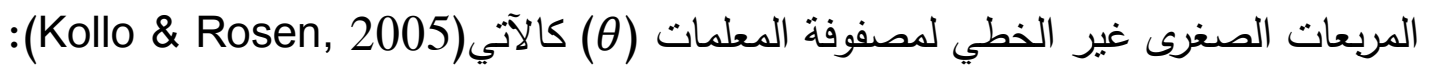




$$
\begin{gathered}
f(\theta)=f(\operatorname{vec}(\theta)) \cong f(\operatorname{vec}(\hat{\theta}))+\left[I_{k} \otimes(\operatorname{vec}(\theta)-\operatorname{vec}(\hat{\theta}))^{\otimes(L-1)}\right]^{\prime} \\
{\left.\left[\frac{\partial f(\operatorname{vec}(\theta))}{\partial \operatorname{vec}^{\prime(\theta)}}\right]^{\prime}\right|_{\theta=\widehat{\theta}}(\operatorname{vec}(\theta)-\operatorname{vec}(\hat{\theta}))}
\end{gathered}
$$

إذ إن

L: تمنل رتبة المشتقة.

وأن

$$
\frac{\partial f(\operatorname{vec}(\theta))}{\partial \operatorname{vec}^{\prime(\theta)}}=\sum_{j=1}^{t-1}\left(y_{t-j}^{\prime} \otimes I_{k}\right)\left[\frac{\partial f\left(\operatorname{vec}\left(\theta^{j}\right)\right)}{\partial \operatorname{vec}^{\prime(\theta)}}\right]^{\prime}
$$

$\frac{\partial f\left(\operatorname{vec}\left(\theta^{j}\right)\right)}{\partial \operatorname{vec}^{\prime}(\theta)}=\sum_{i=1}^{j}\left(\theta^{j-i} \otimes \theta^{i-1}\right)$

إذ إن الطرف الأيمن من المعادلة الأخيرة يمنل مصفوفة ذات سعة (k²xk) .

وبعد تعويض المعادلتين (9) و (10) في المعادلة (8) نحصل على :

$$
\begin{array}{r}
f(\theta)=f(\operatorname{vec}(\theta)) \cong f(\operatorname{vec}(\hat{\theta}))+\left(I_{k} \otimes 1\right)^{\prime} \\
{\left.\left[\sum_{j=1}^{t-1}\left(y_{t-j}^{\prime} \otimes I_{k}\right)\left\{\sum_{i=1}^{j}\left(\theta^{j-i} \otimes \theta^{i-1}\right)\right\}^{\prime}\right]\right|_{\theta=\widehat{\theta}}(\operatorname{vec}(\theta)-\operatorname{vec}(\hat{\theta}))}
\end{array}
$$

$f(\operatorname{vec}(\hat{\theta}))=\sum_{j=1}^{t-1}\left(y_{t-j}^{\prime} \otimes I_{k}\right) \operatorname{vec}\left(\hat{\theta}^{j}\right)$

$D_{t}=\left.\sum_{j=1}^{t-1}\left(y_{t-j}^{\prime} \otimes I_{k}\right)\left\{\sum_{i=1}^{j}\left(\theta^{j-i} \otimes \theta^{i-1}\right)\right\}^{\prime}\right|_{\theta=\widehat{\theta}}$

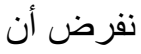

$\therefore f(\theta) \cong f(\operatorname{vec}(\hat{\theta}))-D_{t} \operatorname{vec}(\hat{\theta})+D_{t} \operatorname{vec}(\theta)$

$\cong \underline{c}_{t}+D_{t} \operatorname{vec}(\theta)$

$\underline{c}_{t}=f(\operatorname{vec}(\hat{\theta}))-D_{t} \operatorname{vec}(\hat{\theta})$ 
نعوض المعادلة (12) في المعادلة (5) يصبح أنموذج (1) التقريبي بالثكل الآتي:

$\underline{y}_{t}^{*} \cong \underline{u}_{t}-\left(\underline{c}_{t}+D_{t} \operatorname{vec}(\theta)\right)$

نلاحظ من المعادلة (13) أن نموذج (13) التقريبي أصبح نموذجاً خطياً (الطالب، 2017) . ولنفرض يتوزع توزيع بيسل متعدد المتغيرات المحور المعم ،إذ يمكن إيجاد دالة كثافة

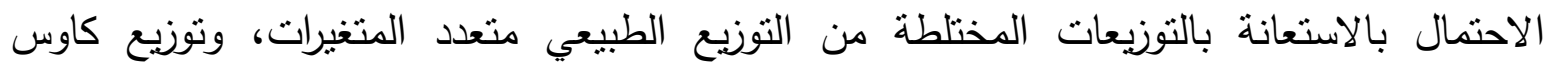

$\underline{u}_{t} \mid \tau \sim N_{k}(\underline{0}, \tau \Sigma)$

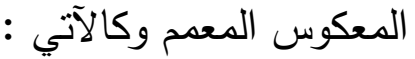

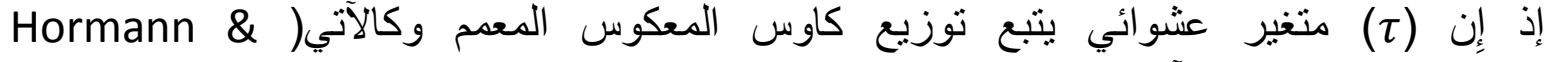
$\tau \sim G I G(\lambda, \psi, v)$

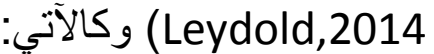

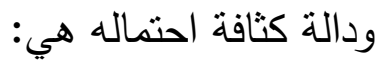

$$
p(\tau)=\frac{\left(\frac{\lambda}{\psi}\right)^{\frac{v}{2}}}{2 \kappa_{v}(\sqrt{\lambda \psi})} \tau^{v-1} \exp \left[-\frac{1}{2}\left\{\left(\frac{\psi}{\tau}\right)+\lambda \tau\right\}\right], \quad \tau>0
$$

إذ إن • (

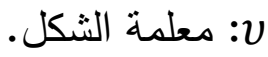

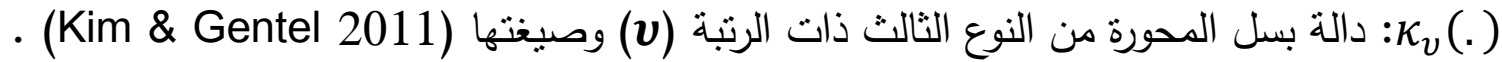
$\kappa_{v}(x)=\frac{1}{2} \int_{0}^{\infty} z^{v-1} \exp \left(-\frac{1}{2} x\left(z+z^{-1}\right)\right) d x$

وإِن دالة كثافة احتمال (

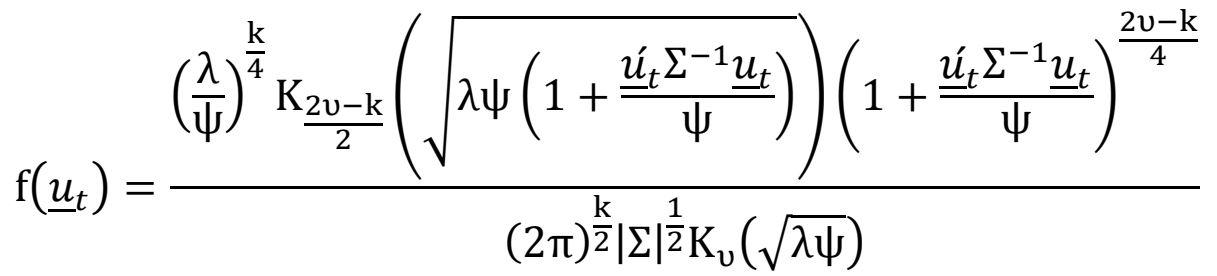

التي تمنل دالة كثافة احتمال توزيع بسل متعدد المتغيرات المحور المعمم ،الذي يوصف بالآتي: $\underline{u}_{t} \sim G M M B_{k}(\underline{0}, \Sigma, \lambda, \Psi, v)$ 


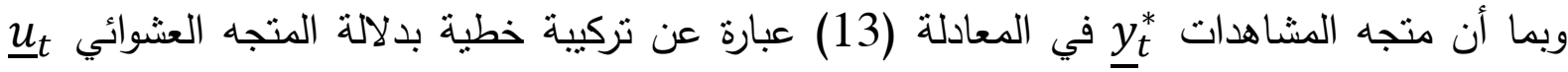

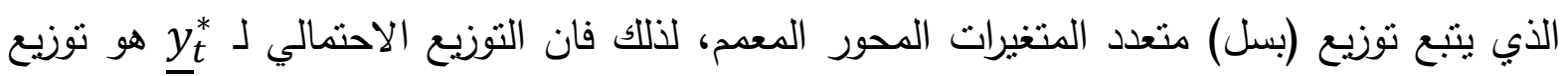
(بسل) متعدد المتغيرات المحور المعدم الذي يوصف بالآتي $\underline{y}_{t}^{*} \sim G M M B_{k}\left(-\left(\underline{c}_{t}+D_{t} \operatorname{vec}(\theta)\right), \Sigma, \lambda, \Psi, v\right)$

التوزيعات الاحتمالية اللاحقة:

يتم في هذا المبحث تقدير معلمات نموذج متجه المتوسطات المتحركة المعرف في المعادلة (13) باستخدام المعلومات السابقة غير الخبرية .

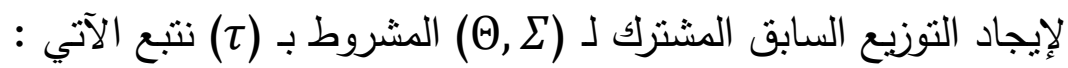
$p(\operatorname{vec}(\theta), \Sigma \mid \tau)=p(\operatorname{vec}(\theta) \mid \Sigma, \tau) p(\Sigma \mid \tau)$ ولإليجاد هذا التوزيع نفرض أن $g_{1}=\operatorname{Ln} L(\operatorname{vec}(\theta), \Sigma \mid \tau)=-\frac{T k}{2} \ln (2 \pi)-\frac{T k}{2} \ln \tau-\frac{T}{2} \ln |\Sigma|-\frac{1}{2 \tau} \operatorname{tr}\left(\Sigma^{-1} w_{1}\right)$ علماً أن

$w_{1}=\sum_{t=1}^{T}\left(\underline{y}_{t}^{*}+\left(\underline{c}_{t}+D_{t} \operatorname{vec}(\theta)\right)\right)\left(\underline{y}_{t}^{*}+\left(\underline{c}_{t}+D_{t} \operatorname{vec}(\theta)\right)\right)^{\prime}$

يتم تفاضل طرفي المعادلة (18)، و نحصل على : $d g_{1}=-\frac{T}{2} \operatorname{tr}\left(\Sigma^{-1} d \Sigma\right)+\frac{1}{2 \tau} \operatorname{tr}\left(\Sigma^{-1} d \Sigma \Sigma^{-1} w_{1}\right)$

$$
\begin{aligned}
& +\frac{1}{2 \tau} \operatorname{tr} \Sigma^{-1}\left[\sum _ { t = 1 } ^ { T } \left(D_{t} \operatorname{dvec}(\theta)\left(\underline{y}_{t}^{*}+\left(\underline{c}_{t}+D_{t} \operatorname{vec}(\theta)\right)\right)^{\prime}\right.\right. \\
& \left.\left.+\left(\underline{y}_{t}^{*}+\left(\underline{c}_{t}+D_{t} \operatorname{vec}(\theta)\right)\right) d \operatorname{vec}^{\prime}(\theta) D_{t}^{\prime}\right)\right]
\end{aligned}
$$

نعيد كتابة الحد الثاني من المعادلة في أعلاه على شكل ثابت ونحصل على (Schott, 1997):

$$
\begin{aligned}
d g_{1}=\frac{1}{2 \tau} \operatorname{tr} & {\left[(d \Sigma) \Sigma^{-1}\left(w_{1}-T \tau \Sigma\right) \Sigma^{-1}\right] } \\
& +\frac{1}{\tau} \sum_{t=1}^{T}\left(\left(\underline{y}_{t}^{*}+\left(\underline{c}_{t}+D_{t} \operatorname{vec}(\theta)\right)\right)^{\prime} \Sigma^{-1} D_{t} \operatorname{dvec}(\theta)\right)
\end{aligned}
$$

وباستخدام خصائص أثز المصفوفة ، وبما أن (ع) هي مصفوفة متتاظرة ذات بعد k × 
$\operatorname{vec}(\Sigma)=D_{k}^{*} V(\Sigma)$

علماً أن * * * مي مصفوفة تستخدم لتعديل عملية تحويل المتجه، وإرجاع أبعاد المصفوفة المحولة إلى $d g_{1}=\frac{1}{2 \tau}\left(\operatorname{vec}(d \Sigma)^{\prime}\right)^{\prime}\left(\Sigma^{-1} \otimes \Sigma^{-1}\right) \operatorname{vec}\left(w_{1}-T \tau \Sigma\right)$

$$
+\frac{1}{\tau} \sum_{t=1}^{T}\left(\left(\underline{y}_{t}^{*}+\left(\underline{c}_{t}+D_{t} \operatorname{vec}(\theta)\right)\right)^{\prime} \Sigma^{-1} D_{t} \operatorname{dvec}(\theta)\right)
$$

$\operatorname{vec}(\mathrm{d} \Sigma)=\operatorname{dvec}(\Sigma)=D_{k}^{*} d V(\Sigma)$

$$
\begin{aligned}
d g_{1}=\frac{1}{2 \tau}( & d V(\Sigma))^{\prime} D_{k}^{* \prime}\left(\Sigma^{-1} \otimes \Sigma^{-1}\right) \operatorname{vec}\left(w_{1}-T \tau \Sigma\right) \\
& +\frac{1}{\tau} \sum_{t=1}^{T}\left(\left(\underline{y}_{t}^{*}+\left(\underline{c}_{t}+D_{t} \operatorname{vec}(\theta)\right)\right)^{\prime} \Sigma^{-1} D_{t} \operatorname{dvec}(\theta)\right)
\end{aligned}
$$

نأخذ التفاضل من المرتبة الثانية للمعادلة(20)

$$
\begin{aligned}
d^{2} g_{1}=\frac{1}{2 \tau}( & d V(\Sigma))^{\prime} D_{k}^{* \prime}\left[d\left(\Sigma^{-1} \otimes \Sigma^{-1}\right)\right] \operatorname{vec}\left(w_{1}-T \tau \Sigma\right) \\
& +\frac{1}{2 \tau}(d V(\Sigma))^{\prime} D_{k}^{* \prime}\left(\Sigma^{-1} \otimes \Sigma^{-1}\right) \operatorname{vec}\left(d w_{1}-T \tau \mathrm{d} \Sigma\right) \\
& -\frac{1}{\tau} \sum_{t=1}^{T} d \operatorname{vec}^{\prime}(\theta) D_{t}^{\prime} \Sigma^{-1} D_{t} \operatorname{dvec}(\theta) \\
& +\frac{1}{\tau} \sum_{t=1}^{T}\left(\left(\underline{y}_{t}^{*}+\left(C_{t}+D_{t} \operatorname{vec}(\theta)\right)\right)^{\prime}\left(d \Sigma^{-1}\right) D_{t} \operatorname{dvec}(\theta)\right)
\end{aligned}
$$

$$
\begin{aligned}
d w_{1}=-\sum_{t=1}^{T} D_{t} \operatorname{dvec}(\theta)\left(\underline{y}_{t}^{*}+\left(\underline{c}_{t}+D_{t} \operatorname{vec}(\theta)\right)\right)^{\prime} \\
-\sum_{t=1}^{T}\left(\left(\underline{y}_{t}^{*}+\left(\underline{c}_{t}+D_{t} \operatorname{vec}(\theta)\right)\right) d \operatorname{vec}^{\prime}(\theta) D_{t}^{\prime}\right)
\end{aligned}
$$


نعوض المعادلة (22) في المعادلة (21)، ونأخذ سالب التوقع الرياضي للطرفين ; نحصل على :

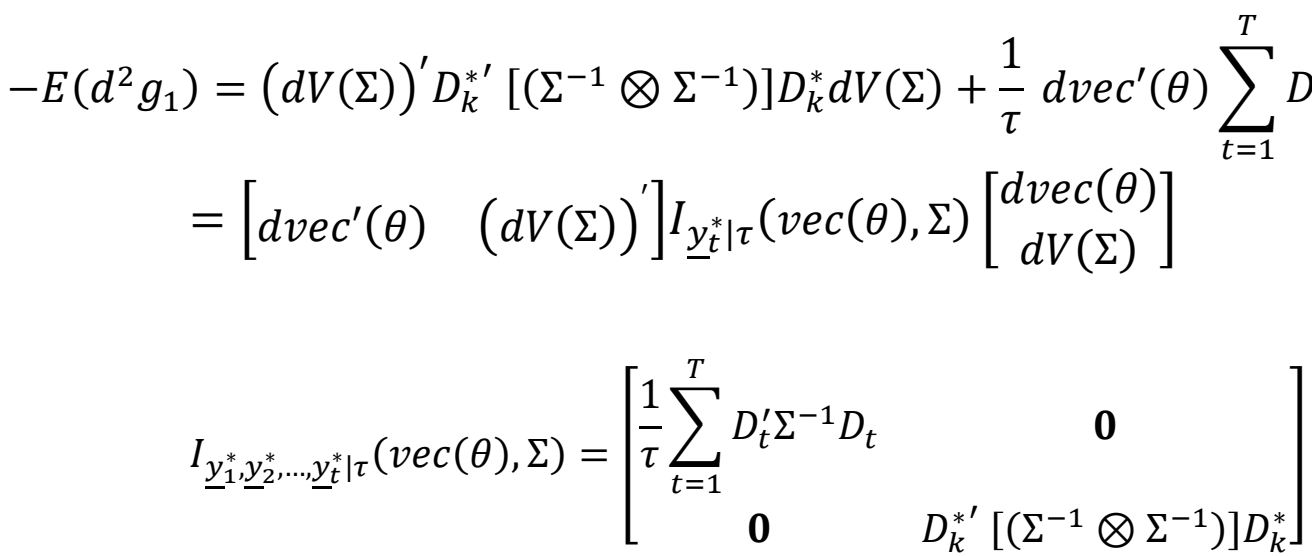

إذ إن

إن مصفوفة المعلومات في أعلاه استتدت على (T) من مشاهدات السلسلة، وبالاعتماد على مشاهدة واحدة فإن المجموع في المصفوفة الموجودة في أول عنصر من القطر الرئيس سوف يحذف، وعليه فإن

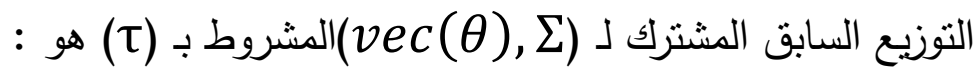

$p(\operatorname{vec}(\theta), \Sigma \mid \tau) \propto\left|I_{\underline{y}_{t}^{*} \mid \tau}(\operatorname{vec}(\theta), \Sigma)\right|^{\frac{1}{2}}$

$p(\operatorname{vec}(\theta), \Sigma \mid \tau) \propto\left|\frac{1}{\tau} D_{t}^{\prime} \Sigma^{-1} D_{t}\right|^{\frac{1}{2}}\left|D_{k}^{*^{\prime}}\left[\left(\Sigma^{-1} \otimes \Sigma^{-1}\right)\right] D_{k}^{*}\right|^{\frac{1}{2}}$

وبما أن D و D D مصفوفات ثابتة لذلك تحذف مع ثابت التتاسب ، عليه يأخذ التوزيع السابق المشترك الصيغة الآتية (الطالب ، 2017):

$p(\operatorname{vec}(\theta), \Sigma \mid \tau) \propto|\Sigma|^{-\frac{1}{2}}|\Sigma|^{-\frac{k}{2}}|\Sigma|^{-\frac{k}{2}}$

$p(\operatorname{vec}(\theta), \Sigma \mid \tau)=|\Sigma|^{-\frac{2 k+1}{2}}$

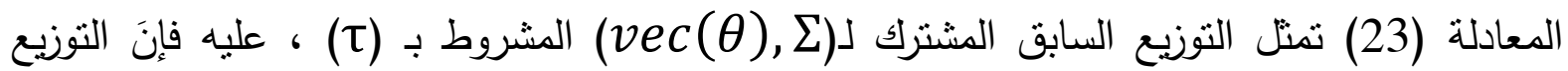

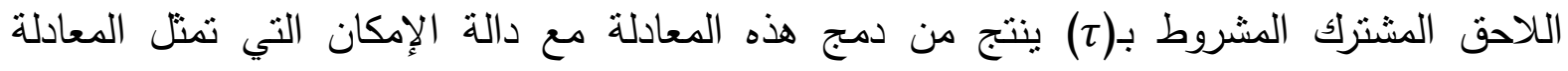

$$
\text { :(24)(الطالب ، 2017) الآتية }
$$

$L(\operatorname{vec}(\theta), \Sigma \mid \tau) \cong \frac{\exp \left[-\frac{1}{2 \tau} \sum_{t=1}^{T}\left(\underline{y}_{t}^{*}-\left[-\left(\underline{c}_{t}+D_{t} \operatorname{vec}(\theta)\right)\right]\right)^{\prime} \Sigma^{-1}\left(\underline{y}_{t}^{*}-\left[-\left(\underline{c}_{t}+D_{t} \operatorname{vec}(\theta)\right)\right]\right)\right]}{(2 \pi)^{\frac{T k}{2}} \tau^{\frac{T k}{2}}|\Sigma|^{\frac{T}{2}}}$

$$
p(\operatorname{vec}(\theta), \Sigma \mid Y, \tau)=p(\operatorname{vec}(\theta), \Sigma \mid \tau) L(\operatorname{vec}(\theta), \Sigma \mid \tau)
$$


$p(\operatorname{vec}(\theta), \Sigma \mid Y, \tau)$

$$
\begin{aligned}
& \propto|\Sigma|^{-\frac{\mathrm{T}+2 \mathrm{k}+1}{2}} \exp \left[-\frac{1}{2 \tau} \sum_{t=1}^{T}\left(\underline{y}_{t}^{*}+\left(\underline{c}_{t}+D_{t} v \operatorname{vec}(\theta)\right)\right)^{\prime} \Sigma^{-1}\left(\underline{y}_{t}^{*}\right.\right. \\
& \left.\left.+\left(\underline{c}_{t}+D_{t} \operatorname{vec}(\theta)\right)\right)\right]
\end{aligned}
$$

بعد إجراء العديد من العطليات الرياضية تصبح المعادلة (25) بالثشكل الآتي :

$p(\operatorname{vec}(\theta), \Sigma \mid Y, \tau) \propto|\Sigma|^{-\frac{\mathrm{T}+2 \mathrm{k}+1}{2}} \exp \left[-\frac{1}{2 \tau} \operatorname{tr}\left(\Sigma^{-1} M_{1}\right)\right]$.

$\exp \left[-\frac{1}{2 \tau} \operatorname{tr}\left(\Sigma^{-1} \sum_{t=1}^{T}\left[D_{t}(\operatorname{vec}(\theta)-\operatorname{vec}(\hat{\theta}))\right]\left[D_{t}(\operatorname{vec}(\theta)-\operatorname{vec}(\hat{\theta}))\right]^{\prime}\right)\right] \ldots$

$M_{1}=\sum_{t=1}^{T}\left(\underline{y}_{t}^{*}+C_{t}\right)\left(\underline{y}_{t}^{*}+C_{t}\right)^{\prime}-\sum_{t=1}^{T}\left(D_{t} \operatorname{vec}(\hat{\theta})\right)\left(D_{t} \operatorname{vec}(\hat{\theta})\right)^{\prime}$ علماً أن 1 معرفة بالآتي

نلحظ أن الجزء الأول من المعادلة (27) يمثل نواة توزيع معكوس وشارت بالمعلمات (T+k) و

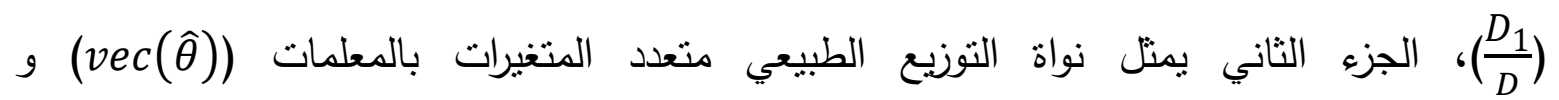
. $\left(\sum D_{t}^{\prime} \Sigma^{-1} D_{t}\right)^{-1}$ ومتوسط التوزيع اللاحق لمتجه المعلمات (vec (v) هو : (vec)

$$
E(\operatorname{vec}(\theta) \mid y))=\operatorname{vec}(\hat{\theta})
$$

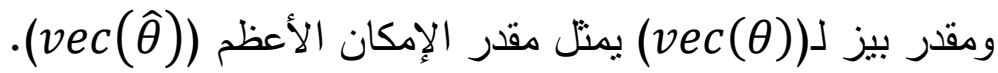

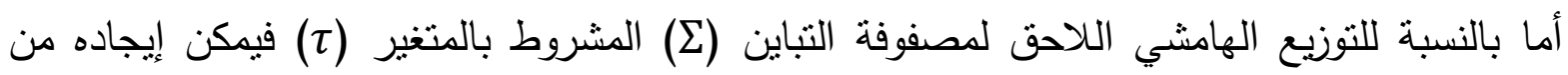

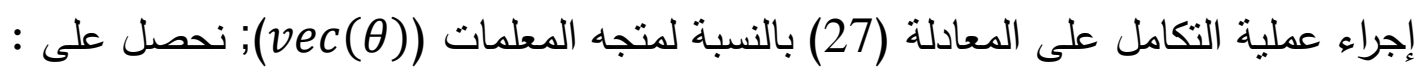
$P(\Sigma \mid Y, \tau) \propto|\Sigma|^{-\frac{T+2 k+1}{2}} \exp \left(-\frac{1}{2 \tau} \operatorname{tr} \Sigma^{-1} M_{1}\right)$

نلحظ أن المعادلة (29) تمنل نواة توزيع Wishart المعكوس بالمعلمات (

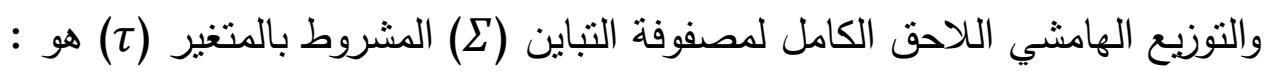




$$
P(\Sigma \mid Y, \tau)=\frac{\left|\frac{M_{1}}{\tau}\right|^{\frac{T+k}{2}}|\Sigma|^{-\frac{T+2 k+1}{2}} \exp \left(-\frac{1}{2 \tau} \operatorname{tr} \Sigma^{-1} M_{1}\right)}{2^{\frac{k(T+k)}{2}} \Gamma_{k}\left(\frac{T+k}{2}\right)}
$$

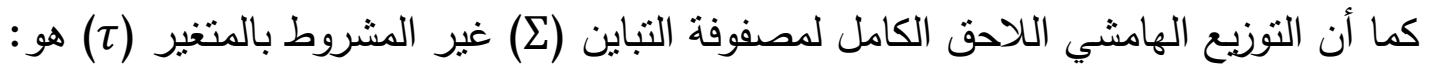
$P(\Sigma \mid Y)=\int_{0}^{\infty} P(\Sigma \mid Y, \tau) P(\tau) d \tau$

$$
\begin{aligned}
& \text { وبتعويض المعادلتين (14) و(30) في المعادلة (31) نحصل على : } \\
& P(\Sigma \mid Y)=\frac{\left|M_{1}\right|^{\frac{T+k}{2}}|\Sigma|^{-\frac{T+2 k+1}{2}}\left(\frac{\lambda}{\psi}\right)^{\frac{k(T+k)}{4}} \mathrm{~K}_{\frac{2 v-k(T+k)}{2}}\left(\sqrt{\lambda \psi\left(1+\frac{\operatorname{tr} \Sigma^{-1} M_{1}}{\psi}\right)}\right)}{2^{\frac{k(T+k)}{2}} \Gamma_{\mathrm{k}}\left(\frac{T+k}{2}\right) \mathrm{K}_{v}(\sqrt{\lambda \psi})\left(1+\frac{\operatorname{tr} \Sigma^{-1} M_{1}}{\psi}\right)^{\frac{2 v-k(T+k)}{4}}}
\end{aligned}
$$

وعليه فإِنَ مقدر بيز هو (الطالب ، 2017)

$$
\widehat{\Sigma}_{B} \cong \frac{M_{1}\left(\frac{\lambda}{\psi}\right)^{\frac{1}{2}} K_{v-1}(\sqrt{\lambda \psi})}{(T-1) K_{v}(\sqrt{\lambda \psi})}
$$

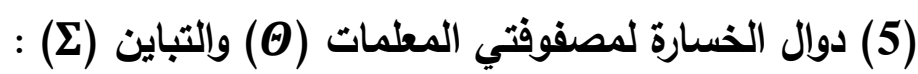
سوف يتم تقدير معلمات أنموذج (1) التقريبي بأُسلوب بيز تحت عدد من دوال الخسارة، وهي :

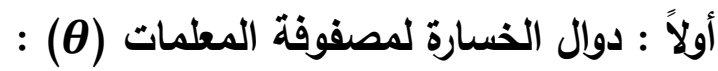
(أ) دوال الخسارة التربيعية: 1 تعرف دالة الخسارة التربيعية بالصيغة الآتية:

$$
\mathrm{L}_{Q}(\hat{\Theta}, \Theta)=\operatorname{tr}\left\{(\hat{\Theta}-\Theta)^{\prime} \mathrm{I}(\hat{\Theta}-\Theta)\right\}
$$

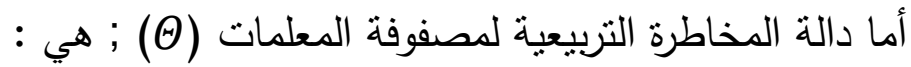




$$
R_{Q}(\widehat{\Theta}, \Theta)=E\left(\mathrm{~L}_{Q}(\widehat{\Theta}, \Theta)\right)=\int_{\Theta} \mathrm{L}_{Q}(\widehat{\Theta}, \Theta) \mathrm{P}(\Theta \mid \mathrm{Y}) \mathrm{d} \Theta
$$

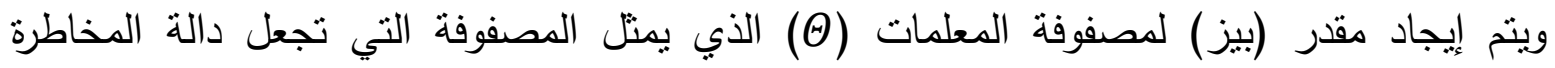
R $R_{Q}(\widehat{\Theta}, \Theta)$ ما يمكن نحصل على:

$$
\therefore \widehat{\Theta}_{\mathrm{Q}}=\mathrm{E}(\Theta \mid \mathrm{Y})
$$

وعليه فإِنَ مقدر (بيز) تحت دالة الخسارة التربيعية يمنل متوسط التوزيع اللاحق •

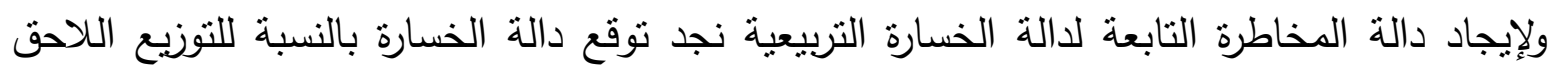

$$
R_{Q}(\hat{\Theta}, \Theta)=\operatorname{tr} E\left\{(\hat{\Theta}-\Theta)^{\prime}(\hat{\Theta}-\Theta)\right\}
$$

نحصل على:

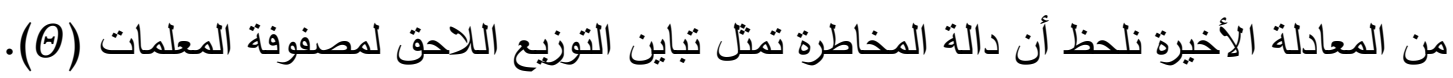

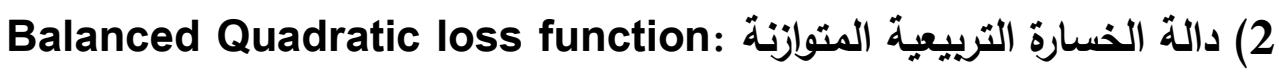
صيغة هذه الدالة هي ( Jozani et al. 2006$)$ $L_{Q B}(\widehat{\Theta}, \Theta)=w \operatorname{tr}\left(\widehat{\Theta}-\Theta_{m}\right)\left(\widehat{\Theta}-\Theta_{m}\right)^{\prime}+(1-w) \operatorname{tr}(\widehat{\Theta}-\Theta)(\widehat{\Theta}-\Theta)^{\prime}$

$$
\begin{array}{r}
\text { وبفرض أن } \Theta_{m} \\
\text { m }=\Theta_{\text {mle }} \text { وعليه نكون دالة الخسارة هي }
\end{array}
$$

$$
\begin{aligned}
\mathrm{L}_{\mathrm{QB}}(\widehat{\Theta}, \Theta)= & w \operatorname{tr}\left(\widehat{\Theta} \widehat{\Theta}^{\prime}-\widehat{\Theta} \Theta_{\mathrm{m}}{ }^{\prime}-\Theta_{\mathrm{m}} \widehat{\Theta}^{\prime}+\Theta_{\mathrm{m}} \Theta_{\mathrm{m}}{ }^{\prime}\right) \\
& +(1-\mathrm{w}) \operatorname{tr}\left(\widehat{\Theta} \widehat{\Theta}^{\prime}-\widehat{\Theta} \Theta^{\prime}-\Theta \widehat{\Theta}^{\prime}+\Theta \Theta^{\prime}\right)
\end{aligned}
$$

لإيجاد مقدر (بيز) نجعل نوقع هذه الدالة بالنسبة لـ @ في نهايته الصغرى ، الذي بعني مخاطرة المقدر

$$
\text { ڤ }
$$

$$
\widehat{\Theta}_{\mathrm{QB}}=\mathrm{w} \Theta_{\mathrm{m}}+(1-\mathrm{w}) \mathrm{E}(\Theta \mid \mathrm{Y})
$$

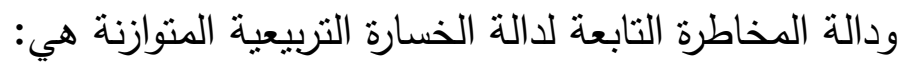

$$
R_{Q B}(\widehat{\Theta}, \Theta)=w \operatorname{tr}\left(\widehat{\Theta}-\Theta_{m}\right)\left(\widehat{\Theta}-\Theta_{m}\right)^{\prime}+(1-w) \operatorname{tr} E\left((\widehat{\Theta}-\Theta)(\widehat{\Theta}-\Theta)^{\prime} \mid Y\right)
$$


هذه الدالة تأخذ صيغة دالة الخسارة التربيعية المتوازنة نفسها، ولكن يضرب الحد الأول منها بوزن

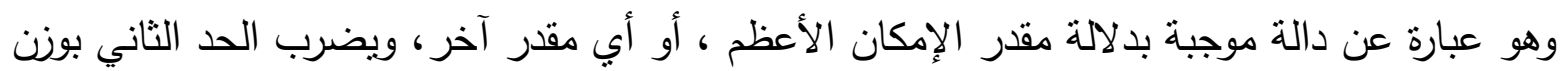

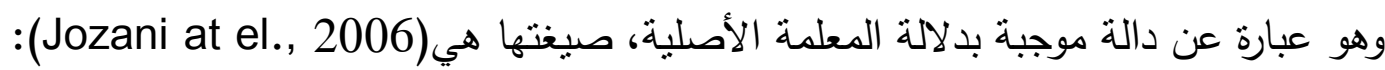

$$
\begin{aligned}
L_{Q W B}(\widehat{\Theta}, \Theta) & =w T\left(\Theta_{m}\right) \operatorname{tr}\left(\widehat{\Theta}-\Theta_{m}\right)\left(\widehat{\Theta}-\Theta_{m}\right)^{\prime} \\
& +(1-w) T(\Theta) \operatorname{tr}(\widehat{\Theta}-\Theta)(\widehat{\Theta}-\Theta)^{\prime}
\end{aligned}
$$

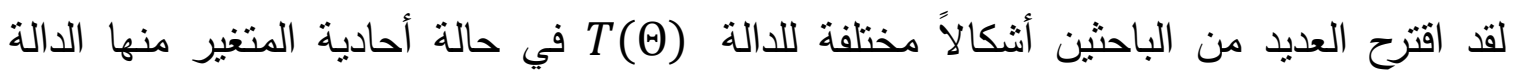
الخطبة الموجبة ، والبعض الآخر استخدم الدالة التي تمثل مربع المعلمة ، أو مقلوب مربع المعلمة أو ... . وبما أن هذه الدالة هي دالة موجبة فقد تم اقتراح الدالة بالثكل الآتي: $T\left(\Theta_{m}\right)=\operatorname{vec}\left(\Theta_{m}\right) \operatorname{vec}\left(\Theta_{m}\right)=\sum_{i=1}^{k} \sum_{j=1}^{k P+1} \Theta_{m(i j)}^{2}$

$T(\Theta)=\operatorname{vec}^{\prime}(\Theta) \operatorname{vec}(\Theta)=\sum_{i=1}^{k} \sum_{j=1}^{k P+1} \Theta_{(i j)}^{2}$

عليه تكون دالة الخسارة بالثكل الآتي :

$$
\begin{aligned}
L_{Q W B}(\widehat{\Theta}, \Theta) & =w \sum_{i=1}^{k} \sum_{j=1}^{k P+1} \theta_{m(i j)}^{2}\left\{\operatorname{tr} \widehat{\Theta} \widehat{\Theta}^{\prime}-2 \operatorname{tr} \widehat{\Theta} \Theta_{\mathrm{m}}^{\prime}+\operatorname{tr} \Theta_{\mathrm{m}} \Theta_{\mathrm{m}}^{\prime}\right\} \\
& +(1-\mathrm{w}) \sum_{i=1}^{k} \sum_{j=1}^{k P+1} \theta_{(i j)}^{2}\left\{\operatorname{tr} \widehat{\Theta} \widehat{\Theta}^{\prime}-2 \operatorname{tr} \widehat{\Theta} \Theta^{\prime}+\operatorname{tr} \Theta \Theta^{\prime}\right\}
\end{aligned}
$$

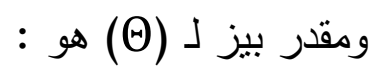

$$
\widehat{\Theta}=\frac{w \sum_{i=1}^{k} \sum_{j=1}^{k P+1} \theta_{m(i j)}^{2} \Theta_{\mathrm{m}}+(1-\mathrm{w}) \mathrm{E}\left(\sum_{i=1}^{k} \sum_{j=1}^{k P+1} \theta_{(i j)}^{2} \Theta \mid Y\right)}{w \sum_{i=1}^{k} \sum_{j=1}^{k P+1} \theta_{m(i j)}^{2}+(1-\mathrm{w}) \mathrm{E}\left(\sum_{i=1}^{k} \sum_{j=1}^{k P+1} \theta_{(i j)}^{2} \mid Y\right)}
$$

ودالة المخاطرة التابعة لدالة الخسارة التربيعية المتوازنة الموزونة هي:

$$
\begin{aligned}
R_{Q W B}(\widehat{\Theta}, \Theta) & =w d \\
& +(1-\mathrm{w})\left[\operatorname{tr} \widehat{\Theta} \widehat{\Theta}^{\prime}\left(\operatorname{tr}(V)+\mu^{\prime} \mu\right)-2 v \operatorname{ec}(\widehat{\Theta}) *\left(\mathrm{M}_{3}+2 V \mu+\left(\operatorname{tr}(V)+\mu^{\prime} \mu\right) \mu\right)\right. \\
& \left.+\left(2 \operatorname{tr}\left(V^{2}\right)+4 \mu^{\prime} V \mu+\left(\operatorname{tr}(V)+\mu^{\prime} \mu\right)^{2}\right)\right]
\end{aligned}
$$


M ر: متجه متوسط التوزيع اللاحق لـ (vec (ب) دوال الخسارة الأُسية الخطية (Linex): 1) دالة الخسارة الأُسية الخطية (Linex):

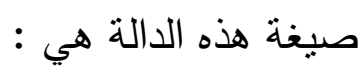

$L_{L}(\widehat{\Theta}, \Theta)=\sum_{i=1}^{k} \sum_{j=1}^{k p+1}\left[\exp \left\{a_{i j}\left(\hat{\theta}_{i j}-\theta_{i j}\right)\right\}-a_{i j}\left(\hat{\theta}_{i j}-\theta_{i j}\right)-1\right]$

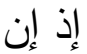

- $\quad i j \neq 0$ : ثابت حقيقي بحيث $a_{i j}$

k : عدد السلاسل المدروسة . لـ

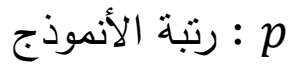

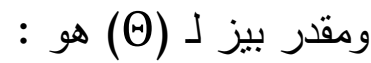

$\therefore \widehat{\theta}_{L(i j)}=-\frac{1}{a_{i j}} \ln E\left(e^{-a_{i j} \theta_{i j}} \mid Y\right)$

ودالة المخاطرة التابعة لدالة الخسارة هي :

$R_{L}(\widehat{\Theta}, \Theta)=\sum_{i=1}^{k} \sum_{j=1}^{k p+1}\left[e^{a_{i j} \widehat{\theta}_{i j}} E\left(e^{-a_{i j} \theta_{i j}} \mid y\right)-a_{i j} \hat{\theta}_{i j}+a_{i j} E\left(\theta_{i j} \mid y\right)-1\right]$

2) دالة الخسارة الأُسية الخطية (Linex) المتوازنة: صيغة هذه الدالة هي :

$$
\begin{aligned}
L_{L B}(\widehat{\Theta}, \Theta)= & \mathrm{w} \sum_{i=1}^{k} \sum_{j=1}^{k p+1}\left[\exp \left\{a_{i j}\left(\hat{\theta}_{i j}-\theta_{m(i j)}\right)\right\}-a_{i j}\left(\hat{\theta}_{i j}-\theta_{m(i j)}\right)-1\right] \\
& +(1-w) \sum_{i=1}^{k} \sum_{j=1}^{k p+1}\left[\exp \left\{a_{i j}\left(\hat{\theta}_{i j}-\theta_{i j}\right)\right\}-a_{i j}\left(\hat{\theta}_{i j}-\theta_{i j}\right)-1\right]
\end{aligned}
$$

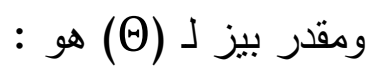

$\therefore \widehat{\theta}_{L B(i j)}=-\frac{1}{a_{i j}} \ln \left[w e^{-a_{i j} \theta_{m(i j)}}+(1-w) E\left(e^{-a_{i j} \theta_{i j}} \mid Y\right)\right]$

ودالة المخاطرة التابعة لدالة الخسارة Linex هي: 


$$
\begin{aligned}
R_{L B}(\widehat{\Theta}, \Theta)= & \mathrm{w} \sum_{i=1}^{k} \sum_{j=1}^{k p+1}\left[\exp \left\{a_{i j}\left(\hat{\theta}_{i j}-\theta_{m(i j)}\right)\right\}-a_{i j}\left(\hat{\theta}_{i j}-\theta_{m(i j)}\right)-1\right] \\
& +(1-w) \sum_{i=1}^{k} \sum_{j=1}^{k p+1}\left[e^{a_{i j} \hat{\theta}_{i j}} E\left(e^{-a_{i j} \theta_{i j}} \mid y\right)-a_{i j} \hat{\theta}_{i j}+a_{i j} E\left(\theta_{i j} \mid y\right)-1\right] \ldots
\end{aligned}
$$

\section{3) دالة الخسارة الأُسية الخطية (Linex) المتوازنة الموزونة:}

هذه الدالة تأخذ صيغة دالة الخسارة الاسية الخطية المتوازنة نفسها لكن يضرب الحد الأول منها

بوزن ويكون هذا الوزن دالة موجبة بدلالة مقدر الإمكان الأعظم ، ويضرب الحد الثاني بوزن وهو عبارة

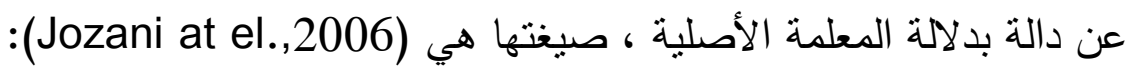

$$
\begin{aligned}
L_{L W B}(\widehat{\Theta}, \Theta)= & w T\left(\Theta_{m}\right) \sum_{i=1}^{k} \sum_{j=1}^{k p+1}\left[\exp \left\{a_{i j}\left(\hat{\theta}_{i j}-\theta_{m(i j)}\right)\right\}-a_{i j}\left(\hat{\theta}_{i j}-\theta_{m(i j)}\right)-1\right] \\
& +(1-w) T(\Theta) \sum_{i=1}^{k} \sum_{j=1}^{k p+1}\left[\exp \left\{a_{i j}\left(\hat{\theta}_{i j}-\theta_{i j}\right)\right\}-a_{i j}\left(\hat{\theta}_{i j}-\theta_{i j}\right)-1\right]
\end{aligned}
$$

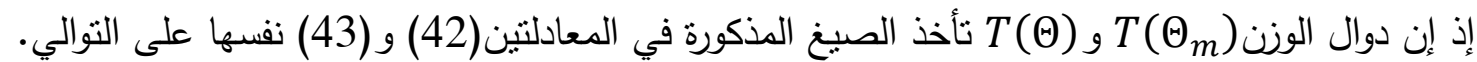

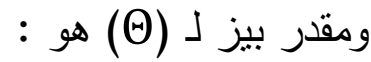

$$
\hat{\theta}_{L W B(i j)}=\frac{1}{a_{i j}} \ln \left[\frac{(1-w) E\left[\operatorname{vec}^{\prime}(\Theta) \operatorname{vec}(\Theta) \mid \mathrm{Y}\right]+\operatorname{wvec}^{\prime}\left(\Theta_{m}\right) \operatorname{vec}\left(\Theta_{m}\right)}{(1-w) E\left[\operatorname{vec}^{\prime}(\Theta) \operatorname{vec}(\Theta) e^{\left.-a_{i j} \theta_{i j} \mid \mathrm{Y}\right]}+\operatorname{wvec}^{\prime}\left(\Theta_{m}\right) \operatorname{vec}\left(\Theta_{m}\right) e^{-a_{i j} \theta_{m(i j)}}\right.}\right] \ldots
$$

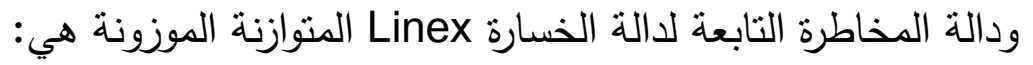

$$
\begin{aligned}
& R(\widehat{\Theta}, \Theta)=\operatorname{vec}^{\prime}\left(\Theta_{m}\right) \operatorname{vec}\left(\Theta_{m}\right) w \sum_{i=1}^{k} \sum_{j=1}^{k p+1}\left[\exp \left\{a_{i j}\left(\hat{\theta}_{i j}-\theta_{m(i j)}\right)\right\}-a_{i j}\left(\hat{\theta}_{i j}-\theta_{m(i j)}\right)-1\right] \\
& +(1-w)\left[\hat{g}(\Theta)+\frac{1}{2} \int_{\tau} \operatorname{vec}^{\prime}(\widehat{B B}) \cdot \operatorname{vec}\left[\frac{\partial^{2} \ln L}{\partial \theta_{f l} \partial \theta_{d r}}\right]^{-1} P(\tau) d \tau\right] \\
& L_{\mathrm{Q}}(\hat{\Sigma}, \Sigma)=\operatorname{tr}\left(\widehat{\Sigma} \Sigma^{-2} \widehat{\Sigma}\right)-2 \operatorname{tr} \widehat{\Sigma} \Sigma^{-1}+k
\end{aligned}
$$

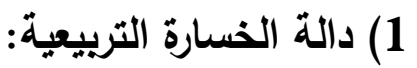




$$
\therefore \widehat{\Sigma}_{\mathrm{Q}}=E\left(\Sigma^{-1} \mid Y\right) *\left[E\left(\Sigma^{-2} \mid Y\right)\right]^{-1}
$$
المعادلة (57) تمثل مقدر (بيز) لمصفوفة التباين (ع) تحت دالة الخسارة التربعية.

$$
\mathrm{R}_{\mathrm{Q}}(\widehat{\Sigma}, \Sigma)=\operatorname{tr}\left(\widehat{\Sigma} \mathrm{E}\left(\Sigma^{-2} \mid \mathrm{Y}\right) \hat{\Sigma}\right)-2 \operatorname{tr} \widehat{\Sigma} \mathrm{E}\left(\Sigma^{-1} \mid \mathrm{Y}\right)+\mathrm{k}
$$
ودالة المخاطرة التربيعية هي: ويتم إيجاد التوقعين غير الثرطيين في المعادلة (58) كالآتي:

$$
E\left(\Sigma^{-1} \mid Y\right)=E_{\tau} E_{\Sigma^{-1}}\left(\Sigma^{-1} \mid Y, \tau\right)
$$

$E\left(\Sigma^{-2} \mid Y\right)=E_{\tau} E_{\Sigma^{-1}}\left(\Sigma^{-2} \mid Y, \tau\right)$

$$
\begin{aligned}
& \Sigma \mid Y, \tau \sim I W_{k}\left(\frac{D}{\tau}, T+k+1\right) \\
& \Sigma^{-1} \mid Y, \tau \sim W\left(\tau D^{-1}, T\right) \\
& \text { وعليه يكون التوقع في المعادلتين (59) و (60) يمثل التوقع لتوزيع وشارت وكالآتي: } \\
& E\left(\Sigma^{-1} \mid Y, \tau\right)=\left(T D^{-1} \tau\right) \\
& E\left(\Sigma^{-1} \mid Y\right)=\left(T D^{-1}\right) * \frac{\kappa_{v+1}(\sqrt{\lambda \psi})}{1} \\
& \kappa_{v}(\sqrt{\lambda \psi})\left(\frac{\lambda}{\psi}\right)^{\frac{1}{2}}
\end{aligned}
$$

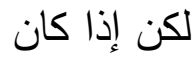

$$
E\left(\Sigma^{-2} \mid Y\right)=\left(\left(\frac{D^{-2}}{(T+1)(T-2)}\right)^{-1}+\left(\frac{D^{-1} \operatorname{tr} D^{-1}}{(T+1)(T-2)}\right)^{-1}\right) * \frac{\kappa_{v+2}(\sqrt{\lambda \psi})}{\kappa_{v}(\sqrt{\lambda \psi})\left(\frac{\lambda}{\psi}\right)}
$$

$$
\begin{aligned}
L_{\mathrm{QB}}(\hat{\Sigma}, \Sigma)= & w \operatorname{tr}\left(\hat{\Sigma} \Sigma_{m}^{-1}-I\right)^{2}+(1-w) \operatorname{tr}\left(\hat{\Sigma} \Sigma^{-1}-I\right)^{2} \\
L_{\mathrm{QB}}(\hat{\Sigma}, \Sigma)=w & \left.\operatorname{tr}\left(\hat{\Sigma} \Sigma_{m}^{-2} \hat{\Sigma}\right)-2 \operatorname{tr}\left(\hat{\Sigma} \Sigma_{m}^{-1}\right)+k\right] \\
& +(1-w)\left[\operatorname{tr}\left(\hat{\Sigma} \Sigma^{-2} \hat{\Sigma}\right)-2 \operatorname{tr}\left(\hat{\Sigma} \Sigma^{-1}\right)+k\right]
\end{aligned}
$$$$
\text { صيغة هذه الدالة هي: - مئ }
$$

$$
\widehat{\Sigma}_{Q B}=\left[w \Sigma_{m}^{-1}+(1-w) E\left(\Sigma^{-1} \mid Y\right)\right] *\left[w \Sigma_{m}^{-2}+(1-w) E\left(\Sigma^{-2} \mid Y\right)\right]^{-1}
$$

و مقدر بيز لـ (ع) هو (الطالب ، 2017): 


$$
\begin{aligned}
& \mathrm{R}_{\mathrm{Q}}(\hat{\Sigma}, \Sigma)=w\left[\operatorname{tr}\left(\hat{\Sigma} \Sigma_{m}^{-2} \hat{\Sigma}\right)-2 \operatorname{tr}\left(\hat{\Sigma} \Sigma_{m}^{-1}\right)+k\right] \\
& +(1-w)\left[\operatorname{tr}\left(\hat{\Sigma} \mathrm{E}\left(\Sigma^{-2} \mid \mathrm{Y}\right) \hat{\Sigma}\right)-2 \operatorname{tr} \hat{\Sigma} \mathrm{E}\left(\Sigma^{-1} \mid \mathrm{Y}\right)+\mathrm{k}\right] \\
& \text { صيغة هذه الدالة هي : } \\
& L_{\mathrm{QWB}}(\hat{\Sigma}, \Sigma)=w T\left(\Sigma_{m}\right) \operatorname{tr}\left(\hat{\Sigma} \Sigma_{m}^{-1}-I\right)^{2}+(1-w) T(\Sigma) \operatorname{tr}\left(\widehat{\Sigma} \Sigma^{-1}-I\right)^{2} \\
& \text { علماً أن (T) (T) و (T) (T) هما دالتا وزن موجبتان وقد اقترحت دالتا الوزن بالثكل الآتي: } \\
& T\left(\Sigma_{m}\right)=\left|\Sigma_{m}\right| \quad, \quad T(\Sigma)=|\Sigma| \\
& L_{\mathrm{QWB}}(\widehat{\Sigma}, \Sigma)=\left|\Sigma_{m}\right|\left[w\left\{\operatorname{tr}\left(\widehat{\Sigma} \Sigma_{m}^{-2} \widehat{\Sigma}\right)-2 \operatorname{tr}\left(\widehat{\Sigma} \Sigma_{m}^{-1}\right)+k\right\}\right] \\
& +|\Sigma|\left[(1-w)\left\{\operatorname{tr}\left(\hat{\Sigma} \mathrm{E}\left(\Sigma^{-2} \mid \mathrm{Y}\right) \hat{\Sigma}\right)-2 \operatorname{tr} \hat{\Sigma} \mathrm{E}\left(\Sigma^{-1} \mid \mathrm{Y}\right)+\mathrm{k}\right\}\right]
\end{aligned}
$$

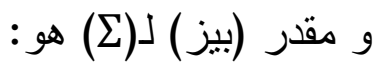

$$
\begin{aligned}
& \widehat{\Sigma}_{\mathrm{QWB}}=\left[\mathrm{w}\left|\Sigma_{m}\right| \Sigma_{m}^{-1}+(1-w) E\left(|\Sigma| \Sigma^{-1} \mid \mathrm{Y}\right)\right] \\
& *\left[w\left|\Sigma_{m}\right| \Sigma_{m}^{-2}+(1-w) E\left(|\Sigma| \Sigma^{-2} \mid \mathrm{Y}\right)\right]^{-1} \\
& \text { ودالة المخاطرة التربيعية المتوازنة الموزونة هي : } \\
& \begin{aligned}
\mathrm{R}_{\mathrm{QW} B}(\widehat{\Sigma}, \Sigma) & =\mathrm{w}\left|\Sigma_{m}\right|\left[\operatorname{tr}\left(\widehat{\Sigma} \Sigma_{m}^{-2} \widehat{\Sigma}\right)-2 \operatorname{tr}\left(\widehat{\Sigma} \Sigma_{m}^{-1}\right)+k\right] \\
& +(1-w)\left\{\operatorname{tr}\left(\hat{\Sigma} \mathrm{E}\left(|\Sigma| \Sigma^{-2} \mid \mathrm{Y}\right) \hat{\Sigma}\right)-2 \operatorname{tr}\left(\widehat{\Sigma} \mathrm{E}\left(|\Sigma| \Sigma^{-1} \mid \mathrm{Y}\right)\right)\right. \\
& +\mathrm{kE}(|\Sigma| \mid \mathrm{Y})\}
\end{aligned} \\
& \text { تكون صيغة هذه الدالة هي : } \\
& L_{\mathrm{E}}(\widehat{\Sigma}, \Sigma)=\operatorname{tr}\left(\hat{\Sigma}^{-1} \Sigma\right)-\ln \left|\hat{\Sigma}^{-1} \Sigma\right|-k
\end{aligned}
$$

k : عدد السلاسل الزمنية المدروسة.

$$
\text { ومقدر (بيز) لـ(I) هو: }
$$

$$
\therefore \widehat{\Sigma}_{E}=\mathrm{E}(\Sigma \mid \mathrm{Y})
$$




$$
R_{E}(\widehat{\Sigma}, \Sigma)=\operatorname{tr}\left(\hat{\Sigma}^{-1} \mathrm{E}(\Sigma \mid \mathrm{Y})\right)-\ln (\widehat{\Sigma})^{-1}-E(\ln (\Sigma) \mid Y)-k
$$

2) دالة الخسارة Entropy المتوازنة :

$$
\begin{aligned}
& L_{\mathrm{E} B}(\widehat{\Sigma}, \Sigma)=w\left[\operatorname{tr}\left(\hat{\Sigma}^{-1} \Sigma_{m}\right)-\ln \left|\hat{\Sigma}^{-1} \Sigma_{m}\right|-k\right] \\
& \begin{aligned}
+ & (1-w)\left[\operatorname{tr}\left(\hat{\Sigma}^{-1} \Sigma\right)-\ln \left|\hat{\Sigma}^{-1} \Sigma\right|-k\right]
\end{aligned} \\
& \text { صيغة هذه الدالة هي : } \\
& \widehat{\Sigma}_{E B}=w \Sigma_{m}+(1-w) \mathrm{E}(\Sigma \mid \mathrm{Y}) \\
& R_{E B}(\hat{\Sigma}, \Sigma)=w\left[\operatorname{tr}\left(\hat{\Sigma}^{-1} \Sigma_{m}\right)-\ln \left(\hat{\Sigma}^{-1} \Sigma_{m}\right)-k\right] \\
& +(1-w)\left[\operatorname{tr}(\widehat{\Sigma} E(\Sigma \mid \mathrm{Y}))-\ln \left(\hat{\Sigma}^{-1}\right)-E(\ln (\Sigma) \mid \mathrm{Y})-k\right]
\end{aligned}
$$

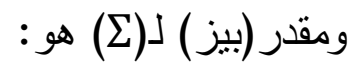

\section{3) دالة الخسارة Entropy المتوازنة الموزونة:}

$$
\begin{aligned}
& \text { صيغة هذه الدالة هي : } \\
& L_{\mathrm{E} W B}(\hat{\Sigma}, \Sigma)=w T\left(\Sigma_{m}\right)\left[\operatorname{tr}\left(\hat{\Sigma}^{-1} \Sigma_{m}\right)-\ln \left|\hat{\Sigma}^{-1} \Sigma_{m}\right|-k\right] \\
& +(1-w) T(\Sigma)\left[\operatorname{tr}\left(\hat{\Sigma}^{-1} \Sigma\right)-\ln \left|\hat{\Sigma}^{-1} \Sigma\right|-k\right] \\
& \text { إذ إن الأوزان ( })
\end{aligned}
$$

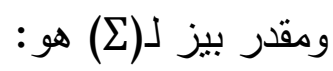

$$
\begin{aligned}
& \widehat{\Sigma}_{E W B}=\left[w\left|\Sigma_{m}\right| \Sigma_{m}+(1-w) \mathrm{E}(|\Sigma| \Sigma \mid \mathrm{Y})\right] *\left[w\left|\Sigma_{m}\right|+(1-w) \mathrm{E}(|\Sigma| \mid \mathrm{Y})\right]^{-1} \ldots \text { (78) } \\
& \text { ودالة المخاطرة Entropy المتوازنة الموزونة هي: } \\
& R_{E W B}(\hat{\Sigma}, \Sigma)=w\left|\Sigma_{m}\right|\left[\operatorname{tr}\left(\hat{\Sigma}^{-1} \Sigma_{m}\right)-\ln \left(\hat{\Sigma}^{-1} \Sigma_{m}\right)-k\right] \\
& +(1-w)\left[\operatorname{tr}\left(\hat{\Sigma}^{-1} \mathrm{E}(|\Sigma| \Sigma \mid \mathrm{Y})\right)\right. \\
& \left.-\ln \left(\hat{\Sigma}^{-1}\right) \mathrm{E}(|\Sigma| \mid \mathrm{Y})-E(|\Sigma| \ln (|\Sigma|) \mid \mathrm{Y})-k \mathrm{E}(|\Sigma| \mid \mathrm{Y})\right] \\
& \text { (6) الجانب التجريبي: }
\end{aligned}
$$

يعرض هذا المبحث تطبيقَ ما توصلنا إليه في المباحث (1، 3،45) ، وذلكت من خلال بيانات مولدة لنِموذج متجه

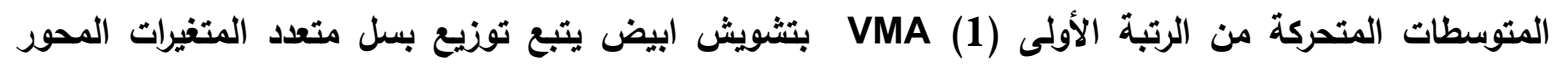

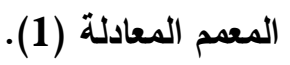


يعد أسلوب المحاكاة (Simulation) من الأساليب الفعالة في توفير قاعدة تجريبية ملائمة للتحقق من الأسلوب المتبع في البحث ، وتأكيداً للقاعدة النظرية قبد الدراسة، نوجد أساليب متعددة للمحاكاة ، وقد شهدت تطوراً كبيراً مواكباً للنطور الذي حصل في استخدام البرمجيات الجاهزة. ويعتمد أسلوب المحاكاة على توليد الأرقام العشوائية التي تحاكي النماذج قيد الدراسة لتوليد بيانات معينة.

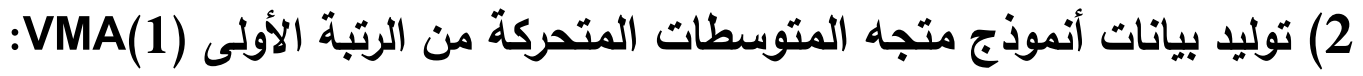
من الصعب نوليد المشاهدات العشوائية لأنموذج متجه المتوسطات المتحركة عندما يكون التشويش الأبيض يتبع توزيع (بسل) متعدد المتغيرات المحور المعمم ، لذلك لجأنا لتوليد هذه المشاهدات عن طريق التوزيعات المختلطة. وتم استخدام التوزيع الطبيعي وتوزيع كاوس المعكوس المعمم كما ذكر لهر سابقاً ـ إذ نم توليد المتجه

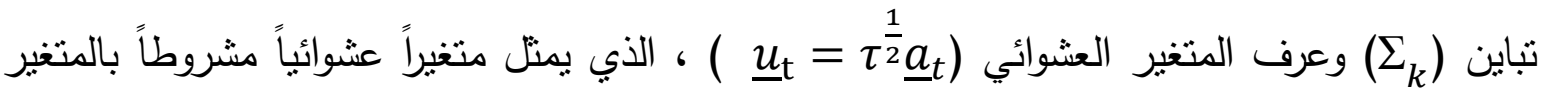

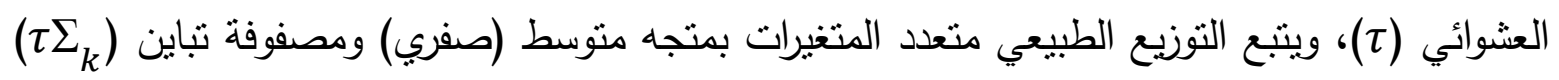

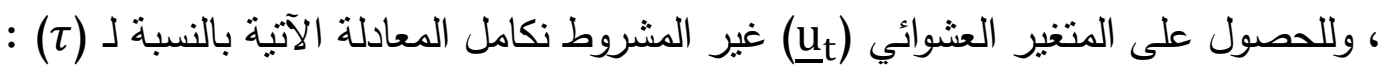
$\underline{u}_{t}=\underline{a}_{t} \int_{0}^{\infty} \tau^{\frac{1}{2}} p(\tau) d \tau$

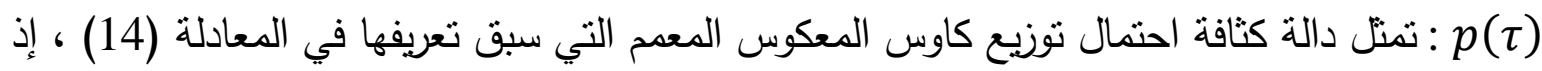

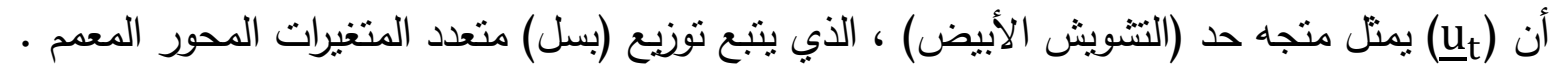

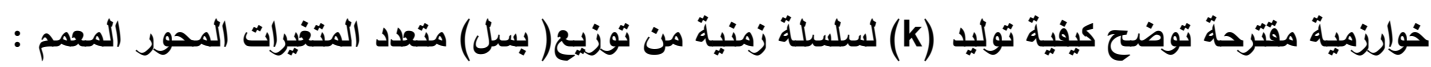

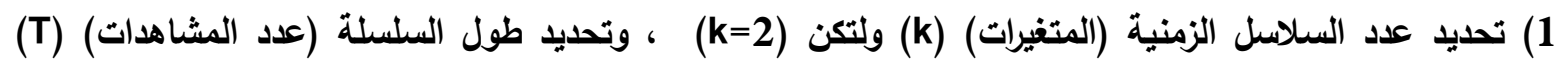
ولنكن (T=100) . (100)

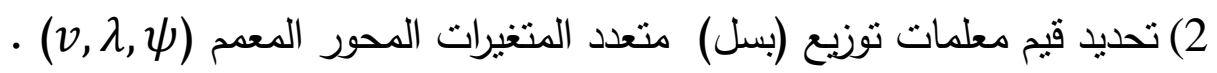
3) تعريف مصفوفة التباين (ع) إذ يجب ان تكون مصفوفة أكيدة الايجابية . 4) توليد (T) مشاهدة عشوائية من التوزيع الطبيعي متعدد المتغيرات وكالآتي:

$$
\underline{a}_{t} \sim N_{k}(\underline{0}, \Sigma) \quad, \quad t=1,2, \ldots, T
$$

5) تحويل المشاهدات المولدة في الخطوة (4) إلى مشاهدات مولدة من توزيع بسل متعدد المتغيرات

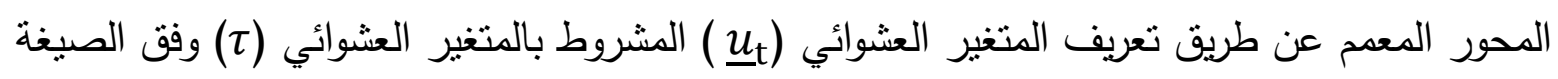
الآتية : $\underline{u}_{t}=\tau^{\frac{1}{2}} \underline{a}_{t}$ 


$$
\underline{a}_{t} \mid \tau \sim N_{k}(\underline{0}, \tau \Sigma)
$$

6) إيجاد المتغير ut غير المشروط بحل المعادلة (80) نحصل على:

$$
\underline{u}_{t}=\underline{a}_{t} \frac{\frac{K_{\frac{2 v+1}{2}}(\sqrt{\lambda \psi})}{K_{v}(\sqrt{\lambda \psi})\left(\frac{\lambda}{\psi}\right)^{\frac{1}{4}}}}{\varepsilon^{2}}
$$

لغرض توليد مشاهدات أنموذج متجه المتوسطات المتحركة من الرتبة الأولى المعرف بالآتي : $\underline{y}_{t}=\underline{c}-\Theta_{1} \underline{a}_{t-1}+\underline{a}_{t}$

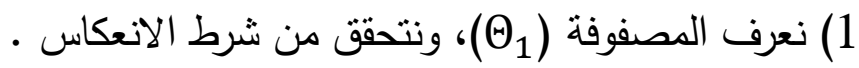
2) نحسب متجهات مشاهدات السلسلة (

$$
\text { المشاهدة (T=100). }
$$

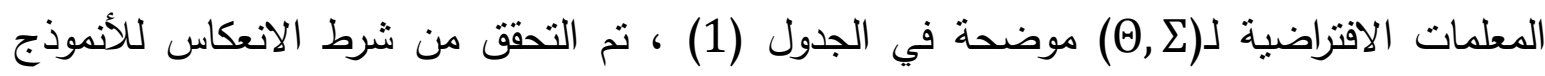
بايجاد جذور المعادلة

$\operatorname{Det}\left(I_{k}+\theta_{1} Z+\theta_{2} Z^{2}+\cdots+\theta_{q} Z^{q 2}\right)=0$

إذ يكون للنموذج خاصية الانعكاس إذا كانت القيمة المطلقة لجذور المعادلة (83) أكبر من الواحد

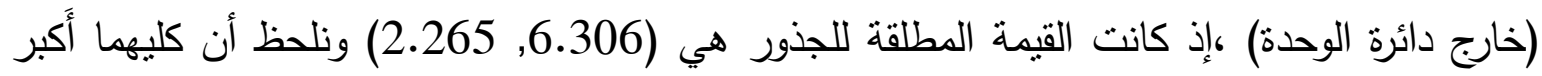
من الواحد مما يدل على تحقق الثرط.

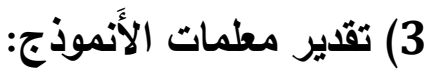

تم تقدير المعلمات (؟, $)$ بأسلوب بيز بآستخدام جميع دوال الخسارة المذكورة ، كما تمت المفاضلة

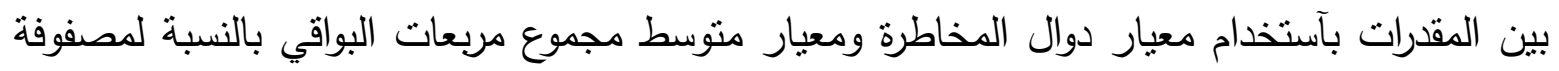

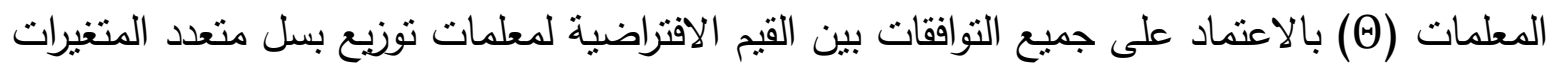
المحور المعمم ،وكذلك الوزن (w) الموضحة في الجدول (1)، وذلك بآستخدام محيط ماتلاب . MATLAB-R2015

\section{الجدول (1) :يوضح القيم الافتراضية المعتمدة لجميع المعلمات}

\begin{tabular}{|c||c||c|c|c||c|}
\hline & $v$ & $\lambda$ & $\psi$ & $w$ & $\theta$ \\
\hline \hline 1 & 3 & 0.2 & 5 & 0.2 & \multicolumn{1}{|c|}{} \\
\hline \hline 2 & 9 & 5 & 9 & 0.5 & \multirow{2}{*}{$\left.\begin{array}{ll}0.2 & 0.1 \\
0.1 & 0.4\end{array}\right]$} \\
\hline \hline 3 & & 9 & & 0.9 & \\
\hline
\end{tabular}


ونتيجة للتوافيق بين جميع القيم الافتراضية لمعلمات توزيع (بسل) متعدد المتغيرات المحور المعمم

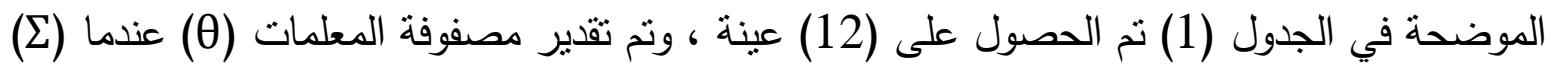
غير معلومة ، ثم المفاضلة بين جميع المقدرات وجميع العينات، وتبين أن أفضل هذه المقدرات هي الموضحة في الجدول (2) (2) (2) الجدول(2) :يوضح دوال المخاطرة ومتوسط مجموع مريعات البواقي لمقدرات بيز لمصفوفة المعلمات (O) تحت

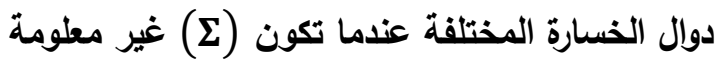

\begin{tabular}{|c|c|c|c|c|}
\hline \multicolumn{5}{|c|}{$v=3, \lambda=9, \psi=5$} \\
\hline $\bar{w}$ & & 0.2 & 0.5 & 0.9 \\
\hline \multirow{2}{*}{$\widehat{\boldsymbol{\theta}}_{\mathbf{Q}}$} & Risk & \multicolumn{3}{|c|}{0.0475} \\
\hline & MSE & \multicolumn{3}{|c|}{2.5390} \\
\hline \multirow{2}{*}{$\widehat{\boldsymbol{\theta}}_{\mathrm{QB}}$} & Risk & 0.0380 & 0.0238 & 0.0048 \\
\hline & MSE & 2.5390 & 2.5390 & 2.5390 \\
\hline \multirow{2}{*}{$\widehat{\boldsymbol{\theta}}_{\mathrm{QWB}}$} & Risk & 0.0062 & 0.0040 & 0.00085072 \\
\hline & MSE & 2.5432 & 2.5410 & 2.5390 \\
\hline \multirow{2}{*}{$\widehat{\boldsymbol{\theta}}_{\mathbf{L}}$} & Risk & \multicolumn{3}{|c|}{0.00024266} \\
\hline & MSE & \multicolumn{3}{|c|}{2.5389} \\
\hline \multirow{2}{*}{$\widehat{\boldsymbol{\theta}}_{\mathrm{LB}}$} & Risk & 0.0001600 & 0.0001213 & 0.00004368 \\
\hline & MSE & 2.5389 & 2.5389 & 2.5389 \\
\hline \multirow{2}{*}{$\widehat{\boldsymbol{\theta}}_{\text {LWB }}$} & Risk & & & \\
\hline & MSE & 2.5623 & 2.5511 & 2.5400 \\
\hline
\end{tabular}

نلحظ أن أفضل المقدرات لدوال الخسارة التربيعية ولدوال خسارة Linex على الترتيب هي الآتي: $\widehat{\boldsymbol{\theta}}_{\mathbf{Q W B}}=\left[\begin{array}{cc}0.1785 & 0.0067 \\ 0.2597 & -0.0007\end{array}\right], \widehat{\boldsymbol{\theta}}_{\mathbf{L B}}=\left[\begin{array}{ll}0.1709 & 0.0114 \\ 0.2516 & 0.0041\end{array}\right], w=0.9$ و المقدر الذي يمتلك أقل MSE، و أقل دالة مخاطرة هو المقدر (7) (الاستنتاجات: *.من نتائج التطبيق العملي تبين أن أفضل مقدر لمتجه معلمات أنموذج المتوسطات المتحركة

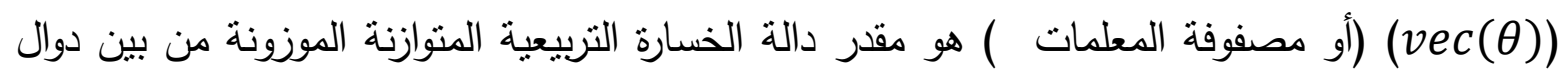
الخسارة التربيعية لجميع العينات المدروسة عند الوزن W=0.9 عند استخدام معيار دالة المخاطرة ، وعند 
استخدام معيار منتسط مجموع مربعات البواقي نلحظ أن المقدر ـق تحت دالة خسارة Linex المنوازنة

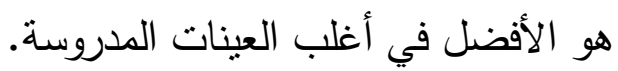
** بثوت المعلمنين ( والعينات. (المصادر: 1) الطالب، محاسن صالح وسعيد،هيفاء عبدالجواد و صالح، وصفي طاهر، (2017)،"التحليل البيزي لبعض نماذج السلاسل الزمنية متعددة المتغيرات غير الطبيعية تحت دوال خسارة مختلفة"، كلية علوم الحاسوب والرياضيات، جامعة الموصل ، أطروحة دكتوراه غير منشورة ،

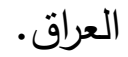

2) العبيدي ، سردد عبدالخالق و سعيد، هيفاء عبدالجواد ، (2013)،"التقدير اللابيزي و البيزي لبعض معلمات نموذج انحدار بسل المحور المعم ميع التطبيق على بيانات سوق العرلق

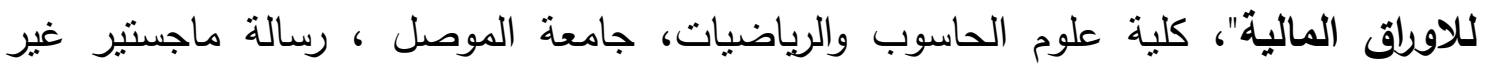

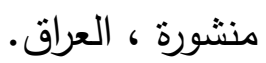

3)Albassam, M. S. and Ali, S. S., (2013), "Selection of prior distribution (1 in the indirect Bayesian identification of Moving average models", Canadian Journal on computing in mathematics, Natural sciences and medicine, Vol. 1. No. 2 .

4) Fan , C. and Yao, S., (2008), "Bayesian approach for ARMA process", International business research, Vol. 4, No. 4.

5) Hormann, W. and Leydold, J., (2014), "Generating generalized Inverse Gaussian Random Variates", Journal Statistics and computing, Vol. 24, Issue 4, pp. 547-557.

6) Jozani, M. J. , Marchand, E. and Parzian, A., (2006)a , "Bayes Estimation Under A General Class of Balanced Loss Functions", https://www.usherbrooke.ca/mathematiques/fileadmin/.../rr36

7) Jozani, M. J., Marchand, E. and Parzian, A., (2006)b , "On Estimation with Weighted Balanced-Type Loss Functions", Statistics \& Probability Letters, 76, pp. 773-780. 
8) Kim, H. and Genton, M. G., (2011), "Characteristic Functions of Scale Mixtures of Multivariate Skew-Normal Distributions", Journal of Multivariate Analysis, 102, pp.1105-1117.

9) Kollo, T. and Rosen, D. V., (2005), "Advance Multivariate Statistics with Matrices", Springer.

10) Malan, K., (2007), "Stationary Multivariate Time Series Analysis", Msc Thesis, University of Pretoria, Pretoria .

11) $\mathrm{Ni}, \mathrm{S}$. and Sun, D. ,(2005), " Bayesian Estimation for Vector Autoregressive Models", American Statistical Association Journal of Business \& Economic Statistics, Vol. 23, No. 1, pp.105-117. 12) Schott, J. R., (1997), "Matrix Analysis for Statistics", John Wiley \& Sons, Canada.

13) Tsay, R. S., (2005),"Analysis of Financial Time Series", $2^{\text {nd }}$ ed., John Wiley \& Sons, Canada.

14) Wei, W.W. S., (2006), "Time Series Analysis: Univariate and Multivariate Methods", $2^{\text {nd }}$ ed., Person Education ,Inc, U.S.A.

15) Weron, R. and Misiorek, A., (2007), "Heavy Tails and Electricity Prices : Do Time Series Models with Non-Gaussian Counterparts ", PRACE NAUKOWE AKADEMII EKONOMCZNEJ WE WROCLAWIU, NR 1076,S. 472-480. 